Open Access

\title{
Improved-high-quality draft genome sequence of Rhodococcus sp. JG-3, a eurypsychrophilic Actinobacteria from Antarctic Dry Valley permafrost
}

\author{
Jacqueline Goordial', Isabelle Raymond-Bouchard', Jennifer Ronholm, Nicole Shapiro², Tanja Woyke², \\ Lyle Whyte $^{1 *}$ and Corien Bakermans ${ }^{3}$
}

\begin{abstract}
The actinobacterium Rhodococcus sp. JG-3 is an aerobic, eurypsychrophilic, soil bacterium isolated from permafrost in the hyper arid Upper Dry Valleys of Antarctica. It is yellow pigmented, gram positive, moderately halotolerant and capable of growth from $30^{\circ} \mathrm{C}$ down to at least $-5^{\circ} \mathrm{C}$. The $5.28 \mathrm{Mb}$ high-quality-draft genome is arranged into 6 scaffolds, containing 9 contigs and 4998 protein coding genes, with 64 \% GC content. Increasing the availability of genome sequences from cold-adapted species is crucial to gaining a better understanding of the molecular traits of cold adaptation in microbes.
\end{abstract}

Keywords: Rhodococcus sp. JG-3, Permafrost, Eurypsychrophile, Dry valleys, Antarctica

\section{Introduction}

Actinobacteria is a ubiquitous phylum in the biosphere, including many environments that exist predominantly and perennially at sub-zero temperatures (cryoenvironments) such as massive ground ice, polar and alpine saline springs and lakes, cryopegs, and permafrost, where it is often a dominant phylum [1]. The molecular traits which allow Actinobacteria to predominate in cryoenvironments remains largely unknown. Actinobacteria may be protected in the permafrost environment by cyst-like resting forms or arthrospores, as observed in Arthrobacter and Micrococcus species isolated from permafrost [2]. It is also possible that dominance of Actinobacteria are due to increased viability and activity in this phylum, as Actinobacteria that can metabolize at sub-zero temperatures have been found [3, 4]. Though Antarctic permafrost has generally been found to harbor orders of magnitude lower culturable microorganisms $\left(0-10^{5}\right.$ cells/g) than Arctic permafrost, Rhodococcus spp. have been readily isolated from both Antarctic and Arctic permafrost [5]. The

\footnotetext{
* Correspondence: Iyle.whyte@mcgill.ca

${ }^{1}$ McGill University, 21,111 Lakeshore Rd., Ste. Anne de Bellevue, QC, Canada, H9X 3V9

Full list of author information is available at the end of the article
}

genome sequence of Rhodococcus sp. JG-3 is also of interest since species within the genus Rhodococcus are known to have versatile degradative metabolisms for recalcitrant xenobiotics [6], including the capability to degrade halogenated organics [7], short and long chain alkanes [8], and petroleum hydrocarbons [9]. Several reports have investigated the catabolic potential of Rhodococcus spp. for contaminant removal at cold temperatures $[8,10,11]$. The public availability of other mesophillic Rhodococcus genomes, in addition to other cryophilic bacterial isolates will enable identification of genes and molecular traits which enable cryophilic organisms like Rhodococcus sp. JG-3 to thrive in cold and extreme environments.

\section{Organism information}

\section{Classification and features}

Rhodococcus sp. JG-3 is a yellow pigmented strain capable of growth from $30{ }^{\circ} \mathrm{C}$ down to at least $-5{ }^{\circ} \mathrm{C}$. It does not require salt, but is moderately halotolerant up to $7 \% \mathrm{NaCl}$. It is a Gram positive short rod (Fig. 1), and grows well on TSB and R2A media. Rhodococcus sp. JG-3 was isolated from University Valley, a small hanging valley (1650-1800 m.a.s.l) above Beacon Valley in the upper elevation McMurdo Dry Valleys, Antarctica. 


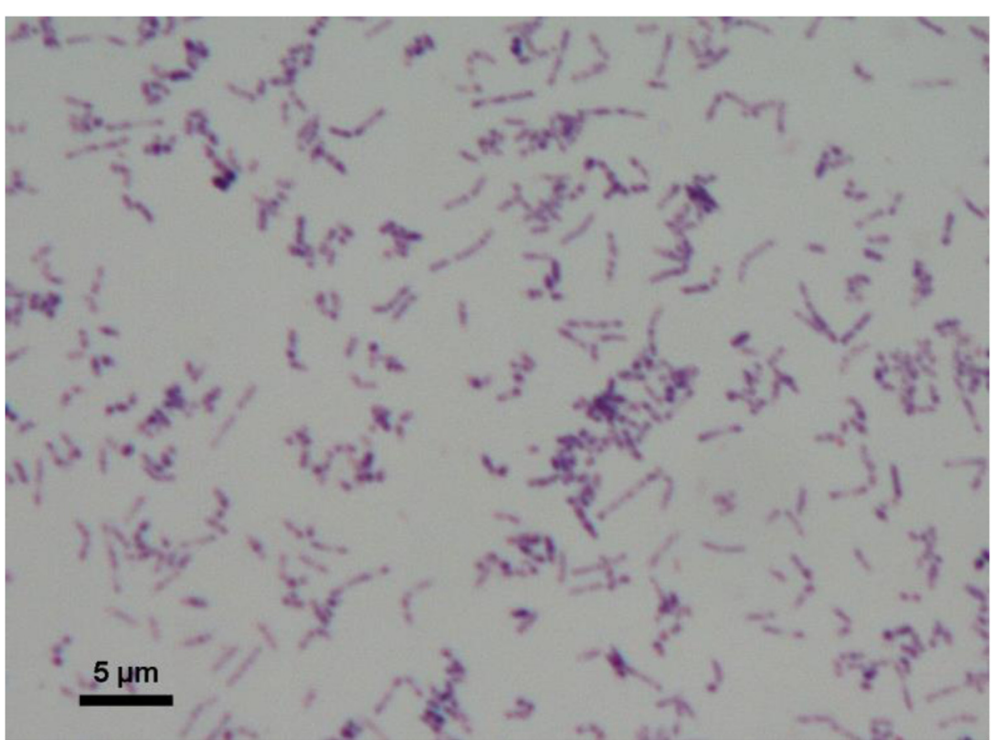

Fig. 1 Gram stain of Rhodococcus JG-3

Table 1 Classification and general features of Rhodococcus sp. JG-3 [13]

\begin{tabular}{|c|c|c|c|}
\hline MIGS ID & Property & Term & Evidence code ${ }^{\mathrm{a}}$ \\
\hline & Classification & Domain Bacteria & TAS [14] \\
\hline & & Phylum Actinobacteria & TAS [15] \\
\hline & & Class Actinobacteria & TAS [15] \\
\hline & & Order Actinomycetales & TAS [14] \\
\hline & & Family Nocardiaceae & TAS [16] \\
\hline & & Genus Rhodococcus & TAS [14] \\
\hline & & Species Rhodococcus & \\
\hline & & Strain JG-3 & \\
\hline & Gram stain & positive & IDA \\
\hline & Cell shape & Rod & IDA \\
\hline & Motility & Not reported & IDA \\
\hline & Sporulation & Not reported & NAS \\
\hline & Temperature range & $<-5^{\circ} \mathrm{C}$ to $30^{\circ} \mathrm{C}$ & NAS \\
\hline & Optimum temperature & $\sim 20^{\circ} \mathrm{C}$ & IDA \\
\hline & $\mathrm{pH}$ range; Optimum & no data; 7 & IDA \\
\hline & Carbon source & R2A, TSA complex media & IDA \\
\hline MIGS-6 & Habitat & Terrestrial, permafrost soil & IDA \\
\hline MIGS-6.3 & Salinity & $0-7 \% \mathrm{NaCl}$ & IDA \\
\hline MIGS-22 & Oxygen requirement & aerobic & IDA \\
\hline MIGS-15 & Biotic relationship & free-living & IDA \\
\hline MIGS-14 & Pathogenicity & Non-pathogen & NAS \\
\hline MIGS-4 & Geographic location & University Valley, Dry Valleys, Antarctica & IDA \\
\hline MIGS-5 & Sample collection & December, 2009 & IDA \\
\hline MIGS-4.1 & Latitude & $77 d 51.817$ s S & IDA \\
\hline MIGS-4.2 & Longitude & $160 \mathrm{~d} 43.524 \mathrm{~s} E$ & IDA \\
\hline MIGS-4.4 & Altitude & $37-42 \mathrm{~cm}$ below soil surface, in ice-cemented permafrost & IDA \\
\hline
\end{tabular}

avidence codes - IDA: Inferred from Direct Assay, TAS: traceable author statement (i.e., a direct report exists in the literature), NAS: Non-traceable Author Statement (i.e., not directly observed for the living, isolated sample, but based on a generally accepted property for the species, or anecdotal evidence). These evidence codes are derived from the Gene Ontology project [17] 
This bacterium was isolated from ice-cemented permafrost soils aged ca. 150,000 years old [12] which experience permanent darkness, hyper oligotrophy ( $0.013 \%$ total carbon), low water activity $(<1 \%$ gravimetric soil moisture content) and constant cold temperature (mean annual soil temperature $-24{ }^{\circ} \mathrm{C}$ ). The classification and general features of Rhodococcus sp. JG-3 are summarized in Table 1.

The 16S rRNA gene sequence of Rhodococcus sp. JG3 was compared using NCBI nucleotide BLAST [18] against the nucleotide collection database (nr/nt) under default parameters, and excluding uncultured microorganisms. Rhodococcus sp. JG-3 showed $99 \%$ similarity to that of $R$. cercidiphylli str. BZ22 [19] (GenBank accession: HQ588861.1), a cold adapted isolate from an industrial site contaminated with heavy oil and heavy metals, and which has demonstrated low temperature degradation of petroleum hydrocarbons [9], and $99 \%$ similarity to Rhodococcus sp. K4-07B (GenBank accession: EF612291) isolated from a semiarid lead-zinc mine tailing site [20]. Phylogenetic analysis based on the 16S rRNA gene of taxonomically classified type strains of the family Nocardiaceae placed Rhodococcus fascians DSM 20669 [21] as the closest validly named species to Rhodococcus sp. JG-3 (Fig. 2). R. fascians DSM 20669 was originally isolated from sweet peas and has an optimum growth temperature of 24 to $27^{\circ} \mathrm{C}$ [21].

\section{Genome sequencing information Genome project history}

Rhodococcus sp. JG-3 was selected for sequencing in 2012 as part of a DOE Joint Genome Institute (JGI) Community Sequencing Program (Quarterly) project to sequence 12 cryophilic isolates from permafrost and cryoenvironments. The Improved Quality Draft assembly and annotation were completed on May 30, 2013. The complete genome sequence of strain JG-3 is available for public access in DDBJ/EMBL/GenBank under accession numbers AXVF01000001- AXVF01000009. The date of Release was December 12, 2013. Table 2 presents the main project information and its association with MIGS version 2.0 compliance [23]. The MIGS record associated with this strain is found in Additional file 1: Table S1.

\section{Growth conditions and DNA isolation}

Rhodococcus JG-3 was grown to stationary phase on TSB medium at room temperature. Genomic DNA was isolated using the Epicentre MasterPure Gram Positive DNA Purification Kit (Epicentre, Madison, Wisconsin) as per the manufacturer's instructions. Purified DNA was evaluated with the NanoDrop 1000 (Thermoscientific, Wilmington, Delaware), according to the standards of the DOE Joint Genome Institute.

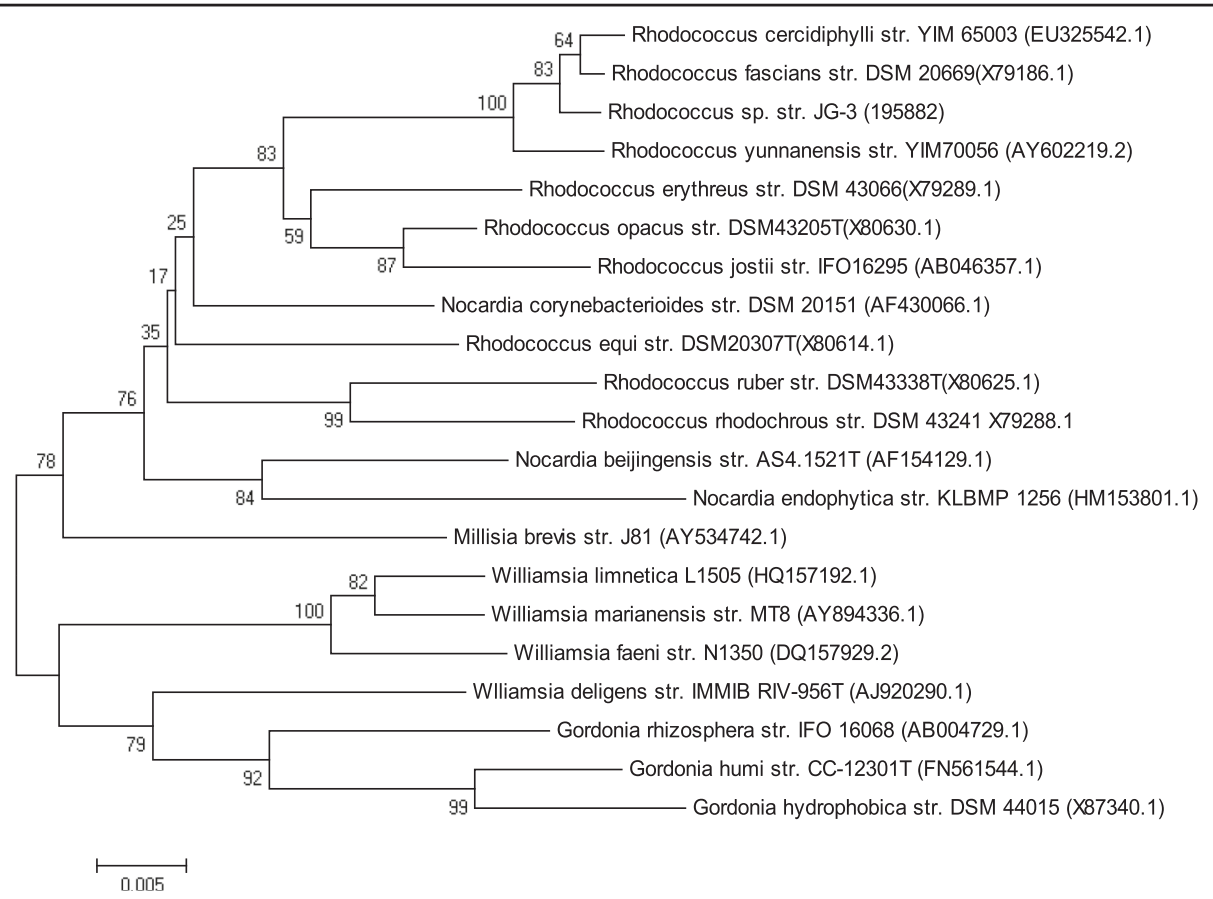

Fig. 2 Phylogenetic tree highlighting the position of Rhodococcus sp. JG-3 relative to selected taxonomically classified strains within the genus Rhodococcus and within the family Nocardiaceae. Phylogenetic inferences were obtained using the neighbor-joining method within MEGA6.05 [22]. Numbers at the nodes are percentages of bootstrap values obtained by repeating the analysis 1,000 times to generate a tree using the maximum composite likelihood model. The GenBank accession numbers for the $16 \mathrm{~S}$ rRNA gene are in parentheses 
Table 2 Project information

\begin{tabular}{|c|c|c|}
\hline MIGS ID & Property & Term \\
\hline MIGS 31 & Finishing quality & Improved-high-quality draft \\
\hline MIGS-28 & Libraries used & Illumina Std. PE, Illumina Clip PE \\
\hline MIGS 29 & Sequencing platforms & Illumina HiSeq 2000 \\
\hline MIGS 31.2 & Fold coverage & 1298.1× Ilumina coverage \\
\hline MIGS 30 & Assemblers & AllpathsLG \\
\hline \multirow[t]{6}{*}{ MIGS 32} & Gene calling method & Prodigal, GenePrimp \\
\hline & Locus Tag & K414 \\
\hline & Genbank ID & AXVF00000000 \\
\hline & $\begin{array}{l}\text { GenBank Date of } \\
\text { Release }\end{array}$ & December 12, 2013 \\
\hline & GOLD ID & Gi22490 \\
\hline & BIOPROJECT & PRJNA195882 \\
\hline \multirow[t]{2}{*}{ MIGS 13} & Source Material Identifier & ARS Culture collection, NRRL: B-65292) \\
\hline & Project relevance & $\begin{array}{l}\text { Permafrost, adaptation to cold, } \\
\text { carbon metabolism }\end{array}$ \\
\hline
\end{tabular}

\section{Genome sequencing and assembly}

The draft genome of Rhodococcus sp. JG-3 was generated at the DOE Joint Genome Institute (JGI) using the Illumina technology. An Illumina std shotgun library and long insert mate pair library was constructed and sequenced using the Illumina HiSeq 2000 platform [24]. 20,820,738 reads totaling 3,123.1 Mb were generated from the std shotgun sequence and 41,292,560 reads totaling 3,757.6 Mb were generated from the long insert mate pair library. All general aspects of library construction and

Table 3 Nucleotide content and gene count levels of the genome

\begin{tabular}{lrc}
\hline Attribute & \multicolumn{1}{l}{ Value } & \% of Total \\
\hline Genome size (bp) & $5,286,918$ & 100.00 \\
DNA coding (bp) & $4,884,848$ & 92.40 \\
DNA G + C (bp) & $3,405,333$ & 64.41 \\
DNA scaffolds & 6 & 100.00 \\
Total genes & 5,067 & 100.00 \\
Protein coding genes & 4,998 & 98.64 \\
RNA genes & 69 & 1.36 \\
Pseudo genes & 60 & 1.18 \\
Genes in internal clusters & NA & \\
Genes with function prediction & 3,977 & 24.18 \\
Genes assigned to COGs & 3,805 & 75.09 \\
Genes with Pfam domains & 4,134 & 81.59 \\
Genes with signal peptides & 370 & 7.30 \\
Genes with transmembrane helices & 1,192 & 23.52 \\
CRISPR repeats & 1 & - \\
\hline
\end{tabular}

sequencing performed at the JGI. All raw Illumina sequence data was passed through DUK, a filtering program developed at JGI, which removes known Illumina sequencing and library preparation artifacts [25]. Filtered Illumina reads were assembled using AllpathsLG (PrepareAllpathsInputs: PHRED 64 $=1$ PLOIDY $=1$ FRAG COVERAGE $=75$ JUMP COVERAGE $=25$; RunAllpathsLG: THREADS $=8$ RUN $=$ std pairs TARGETS $=$ standard VAPI WARN ONLY $=$ True OVERWRITE $=$ True) [26]. The final draft assembly contained 9 contigs in 6 scaffolds. The total size of the genome is $5.3 \mathrm{Mb}$. The final assembly is based on 3,122.6 Mb of Illumina Std PE, 3,757.6 Mb of Illumina CLIP PE post filtered data, which provides an average 1298.1X Illumina coverage of the genome.

\section{Genome annotation}

Genes were identified using Prodigal [27], followed by a round of manual curation using GenePRIMP [28] for

Table 4 Number of genes associated with general COG functional categories

\begin{tabular}{|c|c|c|c|}
\hline Code & Value & $\%$ age & Description \\
\hline J & 176 & 4.17 & $\begin{array}{l}\text { Translation, ribosomal structure } \\
\text { and biogenesis }\end{array}$ \\
\hline A & 1 & 0.02 & RNA processing and modification \\
\hline K & 443 & 10.50 & Transcription \\
\hline$L$ & 173 & 4.10 & Replication, recombination and repair \\
\hline$B$ & 1 & 0.02 & Chromatin structure and dynamics \\
\hline D & 31 & 0.76 & $\begin{array}{l}\text { Cell cycle control, Cell division, } \\
\text { chromosome partitioning }\end{array}$ \\
\hline V & 53 & 1.26 & Defense mechanisms \\
\hline $\mathrm{T}$ & 213 & 5.05 & Signal transduction mechanisms \\
\hline M & 179 & 4.24 & Cell wall/membrane biogenesis \\
\hline $\mathrm{N}$ & 6 & 0.14 & Cell motility \\
\hline$U$ & 43 & 1.02 & Intracellular trafficking and secretion \\
\hline $\mathrm{O}$ & 133 & 3.15 & $\begin{array}{l}\text { Posttranslational modification, } \\
\text { protein turnover, chaperones }\end{array}$ \\
\hline C & 275 & 6.52 & Energy production and conversion \\
\hline G & 288 & 6.83 & Carbohydrate transport and metabolism \\
\hline$E$ & 380 & 9.01 & Amino acid transport and metabolism \\
\hline $\mathrm{F}$ & 97 & 2.3 & Nucleotide transport and metabolism \\
\hline $\mathrm{H}$ & 184 & 4.36 & Coenzyme transport and metabolism \\
\hline I & 233 & 5.52 & Lipid transport and metabolism \\
\hline$P$ & 244 & 5.78 & Inorganic ion transport and metabolism \\
\hline Q & 166 & 3.93 & $\begin{array}{l}\text { Secondary metabolites biosynthesis, } \\
\text { transport and catabolism }\end{array}$ \\
\hline $\mathrm{R}$ & 566 & 13.42 & General function prediction only \\
\hline S & 333 & 7.89 & Function unknown \\
\hline - & 1262 & 24.91 & Not in COGs \\
\hline
\end{tabular}

The total is based on the total number of protein coding genes in the genome 
finished genomes and Draft genomes in fewer than 10 scaffolds. The predicted CDSs were translated and used to search the National Center for Biotechnology Information nonredundant database, UniProt, TIGRFam, Pfam, KEGG, COG, and InterPro databases. The tRNAScanSE tool [29] was used to find tRNA genes, whereas ribosomal RNA genes were found by searches against models of the ribosomal RNA genes built from SILVA [30]. Other non-coding RNAs such as the RNA components of the protein secretion complex and the RNase P were identified by searching the genome for the corresponding Rfam profiles using INFERNAL [17]. Additional gene prediction analysis and manual functional annotation was performed within the Integrated Microbial Genomes platform [1] developed by the Joint Genome Institute, Walnut Creek, CA, USA [31].

\section{Genome properties}

The improved high quality draft genome includes 9 contigs in 6 scaffolds, for a total size of $5286918 \mathrm{bp}, 64.41 \%$ GC content. Most of the genome ( $96 \%, 5092715$ bp) assembled into one scaffold. For the genome, 5067 genes were predicted, 4998 of which are protein-coding genes; 3977 protein coding genes were assigned to a putative function with the remaining annotated as hypothetical proteins. The properties and statistics of the genome are summarized in Tables 3 and 4.

\section{Conclusion}

The genome sequence of Rhodococcus sp. JG-3 will be used for examination of the molecular traits of cold adaptation and to aid understanding of carbon metabolism in cryoenvironments. This is the first reported genome of a bacterium isolated from the Upper Dry Valley permafrost and will provide insight into how microbes survive such extreme conditions. As the availability of genomes from cryophilic strains increases, it may be possible to infer if there is a phylogenetic basis for some cold adaptive traits, as well as identify novel molecular mechanisms for cold adaptation.

\section{Additional file}

Additional file 1: Table S1. Associated MIGS record. (DOC 73 kb)

\section{Competing interests}

The authors declare that they have no competing interests.

\section{Authors' contributions}

Overall development and writing of the manuscript was carried out by JG Isolation of strain was done by JG. Growth and extraction of DNA was carried by JR. Sequencing and annotation of the strain and support was provided by NS, TW IRB, LGW and CB provided critical editing and contributed to the writing of the manuscript.

\section{Acknowledgements}

This work was supported by the NASA ASTEP program and with field support via NSF/OPP (project B-302-M). Support was provided by the Natural Sciences and Engineering Research Council (NSERC) Discovery Grant Program, NSERC Northern Supplements Program, and NSERC CREATE Canadian Astrobiology Training Program (CATP). The work conducted by the U.S. Department of Energy Joint Genome Institute is supported by the Office of Science of the U.S Department of Energy under Contract No. DE-AC02-05CH11231. Additional thanks to Marcel Huntemann, James Han, Amy Chen, Nikos Kyrpides, Victor Markowitz, Krishna Palaniappan, Natalia Ivanova, Natalia Mikhailova, Galina Ovchinnikova, Andrew Schaumberg, Amrita Pati, Dimitrios Stamatis, Tatiparthi Reddy, Henrik P. Nordberg, Michael N. Cantor, and Susan X. Hua of JGI.

\section{Author details}

McGill University, 21,111 Lakeshore Rd., Ste. Anne de Bellevue, QC, Canada, H9X 3V9. ${ }^{2}$ DOE Joint Genome Institute, Walnut Creek, CA, USA. ${ }^{3}$ Altoona College, Pennsylvania State University, Altoona, PA, USA.

Received: 16 September 2014 Accepted: 17 June 2015

Published online: 03 September 2015

\section{References}

1. Goordial J, Lamarche-Gagnon G, Lay C-Y, Whyte L. Left Out in the Cold: Life in Cryoenvironments. Polyextremophiles: Springer; 2013. p. 335-63.

2. Soina VS, Mulyukin AL, Demkina EV, Vorobyova EA, El-Registan GI. The structure of resting bacterial populations in soil and subsoil permafrost. Astrobiology. 2004;4(3):345-58.

3. Bottos EM, Vincent WF, Greer CW, Whyte LG. Prokaryotic diversity of arctic ice shelf microbial mats. Environmental microbiology. 2008;10(4):950-66.

4. Tuorto SJ, Darias P, McGuinness LR, Panikov N, Zhang T, Häggblom MM, et al. Bacterial genome replication at subzero temperatures in permafrost. The ISME journal. 2014;8(1):139-49.

5. Goordial J, Whyte L. Microbial Life in Antarctic Permafrost Environments. Antarctic Terrestrial Microbiology: Springer Berlin Heidelberg; 2014. p. 217-32.

6. Larkin MJ, Kulakov LA, Allen CC. Biodegradation and Rhodococcus-masters of catabolic versatility. Current Opinion in Biotechnology. 2005;16(3):282-90.

7. Haeggblom M, Salkinoja-Salonen M. Biodegradability of chlorinated organic compounds in pulp bleaching effluents. Water Science \& Technology. 1991;24(3-4):161-70

8. Whyte LG, Hawari J, Zhou E, Bourbonnière L, Inniss WE, Greer CW. Biodegradation of Variable-Chain-Length Alkanes at Low Temperatures by a Psychrotrophic Rhodococcussp. Applied and environmental microbiology. 1998;64(7):2578-84.

9. Margesin R, Moertelmaier C, Mair J. Low-temperature biodegradation of petroleum hydrocarbons ( $\mathrm{n}$-alkanes, phenol, anthracene, pyrene) by four actinobacterial strains. International Biodeterioration \& Biodegradation 2012.

10. Whyte L, Slagman S, Pietrantonio F, Bourbonniere L, Koval S, Lawrence J, et al. Physiological adaptations involved in alkane assimilation at a low temperature by Rhodococcus sp. strain Q15. Applied and environmental microbiology. 1999:65(7):2961-8.

11. Ruberto LA, Vazquez S, Lobalbo A, Mac CW. Psychrotolerant hydrocarbon-degrading Rhodococcus strains isolated from polluted Antarctic soils. Antarctic Science. 2005;17(01):47-56.

12. Lacelle D, Davila AF, Fisher D, Pollard WH, DeWitt R, Heldmann J, et al. Excess ground ice of condensation-diffusion origin in University Valley, Dry Valleys of Antarctica: Evidence from isotope geochemistry and numerical modeling. Geochimica et Cosmochimica Acta. 2013;120:280-97.

13. Field D, Garrity G, Gray T, Morrison N, Selengut J, Sterk P, et al. The minimum information about a genome sequence (MIGS) specification. Nature biotechnology. 2008;26(5):541-7.

14. Skerman VBD, McGowan V, Sneath PHA. Approved lists of bacterial names. International Journal of Systematic Bacteriology. 1980;30(1):225-30.

15. Stackebrandt E, Rainey FA, Ward-Rainey NL. Proposal for a new hierarchic classification system, Actinobacteria classis nov. International journal of systematic bacteriology. 1997:47(2):479-91.

16. Castellani A, Chalmers A. Family Nocardiaceae. Manual of Tropical Medicine. Thirdth ed. New York: Williams, Wood and Co; 1919.

17. Ashburner M, Ball CA, Blake JA, Botstein D, Butler H, Cherry JM, et al. Gene Ontology: tool for the unification of biology. Nature genetics. 2000;25(1):25-9. 
18. Altschul SF, Gish W, Miller W, Myers EW, Lipman DJ. Basic local alignment search tool. Journal of molecular biology. 1990;215(3):403-10.

19. Li J, Zhao G-Z, Chen H-H, Qin S, Xu L-H, Jiang C-L, et al. < i > Rhodococcus cercidiphylli</i $>$ sp. nov., a new endophytic actinobacterium isolated from $\mathrm{a}<\mathrm{i}>$ Cercidiphyllum japonicum $</ \mathrm{i}>$ leaf. Systematic and applied microbiology. 2008;31(2):108-13.

20. Mendez MO, Neilson JW, Maier RM. Characterization of a bacterial community in an abandoned semiarid lead-zinc mine tailing site. Applied and environmental microbiology. 2008;74(12):3899-907.

21. Goodfellow M. Reclassification of Corynebacterium fascians (Tilford) Dowson in the Genus Rhodococcus as Rhodococcus fascians comb. nov. Systematic and Applied Microbiology. 1984;5(2):225-9.

22. Tamura K, Stecher G, Peterson D, Filipski A, Kumar S. MEGA6: Molecular Evolutionary Genetics Analysis Version 6.0. Molecular biology and evolution. 2013;30(12):2725-9.

23. Field D, Garrity G, Gray T, Morrison N, Selengut J, Sterk P, et al. The minimum information about a genome sequence (MIGS) specification. Nat Biotechnol. 2008;26(5):541-7.

24. Bennett S. Solexa Itd. Pharmacogenomics. 2004;5(4):433-8.

25. Mingkun L CA, Han J. DUK. unpublished 2011.

26. Gnerre S, MacCallum I, Przybylski D, Ribeiro FJ, Burton JN, Walker BJ, et al. High-quality draft assemblies of mammalian genomes from massively parallel sequence data. Proceedings of the National Academy of Sciences. 2011;108(4):1513-8.

27. Hyatt D, Chen G-L, LoCascio P, Land M, Larimer F, Hauser L. Prodigal: prokaryotic gene recognition and translation initiation site identification. BMC bioinformatics. 2010;11(1):119.

28. Pati A, Ivanova NN, Mikhailova N, Ovchinnikova G, Hooper SD, Lykidis A, et al. GenePRIMP: a gene prediction improvement pipeline for prokaryotic genomes. Nature methods. 2010;7(6):455-7.

29. Lowe TM, Eddy SR. tRNAscan-SE: a program for improved detection of transfer RNA genes in genomic sequence. Nucleic acids research. 1997;25(5):0955-964.

30. Pruesse E, Quast C, Knittel K, Fuchs BM, Ludwig W, Peplies J, et al. SILVA: a comprehensive online resource for quality checked and aligned ribosomal RNA sequence data compatible with ARB. Nucleic acids research. 2007;35(21):7188-96.

31. Markowitz VM, Mavromatis K, Ivanova NN, Chen I-MA, Chu K, Kyrpides NC. IMG ER: a system for microbial genome annotation expert review and curation. Bioinformatics. 2009;25(17):2271-8.

\section{Submit your next manuscript to BioMed Central and take full advantage of:}

- Convenient online submission

- Thorough peer review

- No space constraints or color figure charges

- Immediate publication on acceptance

- Inclusion in PubMed, CAS, Scopus and Google Scholar

- Research which is freely available for redistribution 